\title{
Endocarditis caused by an oral taxon species of Bergeyella identified by partial 16S rDNA sequencing: case report and review of the literature
}

\author{
Lauren Clark MD ${ }^{1}$, Michael D Parkins MD, FRCPC 1 , Barbara L Chow BSc ${ }^{2}$, Tarah Lynch PhD², \\ Deirdre Church MD, PhD, FRCPC $1,2,3$
}

Bergeyella spp bacteremia is a rare cause of infective endocarditis and is typically associated with animal contact. This case report presents a case of culture-negative endocarditis caused by Bergeyella spp oral taxon 422 in a 49-year-old man with severe periodontal disease but no animal contact. Multiple sets of blood cultures were negative, but broad-range 16S rDNA polymerase chain reaction (PCR) amplification and sequencing repeatedly detected this organism in the patient's bloodstream. Empiric broad-spectrum antibiotic treatment against Bergeyella spp resulted in resolution of clinical symptoms, resolution of bloodstream infection, and cure. This is the first human case of endocarditis caused by an oral-associated species of Bergeyella described in the literature. Culture-negative endocarditis due to Bergeyella spp from severe periodontal disease may be missed unless molecular detection methods are used.

KEY WORDS: endocarditis; Bergeyella spp; periodontal disease; broad-range 16S PCR

La bactériémie causée par des espèces de Bergeyella est une rare cause d'endocardite infectieuse qui s'associe habituellement au contact avec des animaux. Le présent rapport de cas décrit une endocardite négative à la culture causée par le taxon oral 422 des espèces de Bergeyella chez un homme de 49 ans ayant une parodontopathie marquée, mais qui n'avait pas eu de contacts avec des animaux. De nombreuses analyses sanguines sont revenues négatives, mais l'amplification en chaîne par polymérase $(\mathrm{PCR})$ et le séquencÇage de l'ADNr 16s à large spectre ont décelé cet organisme à répétition dans le sang du patient. Une antibiothérapie empirique à large spectre contre les espèces de Bergeyella a favorisé la résolution des symptômes cliniques et de l'infection sanguine et guéri le patient. II s'agit du premier cas d'endocardite humaine causée par des espèces de Bergeyella d'origine buccale à être décrit dans une publication. On risque de passer à côté d'une endocardite négative en culture causée par des espèces de Bergeyella provenant d'une parodontopathie marquée, à moins de recourir à des techniques moléculaires de dépistage.

MOTS-CLÉS : endocardite, espèces de Bergeyella, parodontopathie, PCR $16 \mathrm{~S}$ à large spectre

${ }^{1}$ Department of Medicine, University of Calgary, Calgary, Alberta; ${ }^{2}$ Calgary Laboratory Services, Calgary, Alberta; ${ }^{3}$ Department of Pathology \& Laboratory Medicine, University of Calgary, Calgary, Alberta

Correspondence: Deirdre Church, c/o Calgary Laboratory Services, Calgary, Alberta T2L 2K8. Telephone: 403-770-3281.

Email: Deirdre.church@cls.ab.ca

\section{CASE PRESENTATION}

A 49-year-old Canadian man presented with a 2-month history of recurrent night sweats, palpitations, and chest pain to the Foothills Medical Centre in Calgary, Alberta.
He had extensive dental caries with non-restorable tooth decay and severe posterior molar bone loss. Three years earlier, he had been diagnosed with native aortic valve endocarditis caused by Streptococcus viridans group and received 6 weeks of parenteral antibiotics (limited details

\section{OPEN

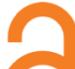 \\ ACCESS CC BY-NC}

Official Journal of the Association of Medical Microbiology and Infectious Disease Canada 2.1, 2017 doi:10.3138/jammi.2.008 
are available because he was treated in another health care jurisdiction). His medical history was otherwise significant for congenital bicuspid aortic valve, cigarette smoking, and poor dentition. He gave no history of injection drug use. Hepatitis A, B, and C and HIV serology tests were negative.

The patient was afebrile on admission. Resting heart rate was $58 \mathrm{bpm}$, blood pressure was $114 / 59 \mathrm{mmHg}$, and oxygen saturation $\left(\mathrm{SpO}_{2}\right)$ was $94 \%-99 \%$ while breathing ambient air. A 2/6 systolic murmur was the only peripheral manifestation of endocarditis. White blood cell (WBC) count was not elevated at $6.2 \times 10^{9} / \mathrm{L}$ (69\% neutrophils). The patient had not recently received antibacterials. Despite several blood cultures ( 3 sets each consisting of an aerobic/anaerobic bottle pair), no pathogen was detected after 5 days of continuous incubation in a BacTAlert 3D system (bioMérieux) (see Figure 1). A transesophageal echocardiogram (TEE) revealed aortic and mitral valve vegetations along with severe aortic insufficiency. Empiric broad-spectrum antibiotic treatment with parenteral ceftriaxone $(2 \mathrm{~g}$ every 24 hours and vancomycin $1 \mathrm{~g}$ every 12 hours (target trough of $15-20 \mu \mathrm{g} / \mathrm{mL}$ ) was immediately started.

The patient was assessed by the cardiovascular surgical team and advanced for semi-urgent operative management. Dentistry was consulted because of his severe periodontal disease, and 10 teeth were extracted on the day before cardiovascular surgery. Aortic valve reconstruction requiring a bovine pericardial patch and debridement of the anterior mitral valve leaflet vegetation were performed without intra-operative complications. Aortic valve tissue cultures were negative for both bacterial and fungal pathogens.

Once clinically stable after surgery, the patient was discharged home to receive a prolonged course of parenteral antibiotic therapy with the same empiric regimen. However, he returned to the hospital 4 days after discharge with new onset fatigue, subjective fevers, chills, and dizziness. He was hypotensive, with a systolic blood pressure of only $50 \mathrm{mmHg}$. He was afebrile, with no evidence of ongoing cardiac or focal infection, and WBC count was not elevated. A large pericardial effusion was demonstrated by transthoracic echocardiogram (TTE), and the patient was admitted to the coronary care unit for treatment of tamponade with secondary cardiogenic shock. Pericardiocentesis was performed to drain $600 \mathrm{~mL}$ of serosanguineous fluid. The fluid had a normal $\mathrm{pH}$ (1) and glucose $(3.3 \mathrm{mmol} / \mathrm{L})$. WBC count was also normal $\left(3.4 \times 10^{9} / \mathrm{L}\right.$, with $33 \%$ neutrophils and $48 \%$ lymphocytes). Fluid cultures were negative for bacterial, fungal, and mycobacterial pathogens. Q fever serology, serum and pericardial fluid polymerase chain reaction (PCR) for Bartonella, and repeat blood cultures were also negative. Repeat TTE the next day confirmed no re-collection of pericardial fluid. There was no change to the patient's antibiotic regimen because there was no evidence of ongoing infection. He was discharged 2 days later with complete resolution of his presenting symptoms and continued outpatient antibiotic therapy. He received a total of 6 weeks of ceftriaxone ( $2 \mathrm{~g}$ every 24 hours) and vancomycin (adjusted to $1 \mathrm{~g}$ every 24 hours), with regular monitoring of trough vancomycin levels (target of $15-20 \mu \mathrm{g} / \mathrm{mL}$ ).

Persistent night sweats began again 2 weeks after stopping parenteral antibiotics. Markers of inflammation, including WBC count $\left(5.4 \times 10^{9} / \mathrm{L}\right)$, C-reactive protein $(\mathrm{CRP})$ $(1.4 \mathrm{mg} / \mathrm{L})$, and erythrocyte sedimentation rate (ESR) $(17 \mathrm{~mm} / \mathrm{h})$, were within normal limits. A repeat TEE showed no new changes to either heart valve. A computed tomography (CT) scan of the chest and a nuclear medicine bone scan focused on the pericardial aortic patch/valve were also unremarkable. Repeat blood cultures 1 month apart again failed to detect any organism. The patient was followed closely, but, given the absence of either echocardiographic or microbiologic evidence of relapsed/ recurrent infection, empiric antibiotic treatment was not initiated. His symptoms continued for 2 months despite persistently negative blood cultures. This prompted broadrange 16S PCR and sequencing on multiple prospectively and retrospectively collected blood samples. Molecular testing on multiple sequentially collected blood samples run on different days showed infection with Bergeyella spp, most closely related to oral taxon 422 (2). Broadspectrum antibiotic therapy was again started, including both of the drugs given during the previous course (ceftriaxone and vancomycin), plus daily high-dose gentamicin. Clinical symptoms quickly resolved on this regimen, which was continued for another 6-week course. Clinical cure was demonstrated by repeatedly negative broad-range 16S PCR tests on follow-up blood samples.

\section{Laboratory methods}

Multiple blood cultures were processed using the BacT/ Alert system. Each blood culture set included both an FA (aerobic) and an FN (anaerobic) bottle. Blood cultures that were negative after the routine 5-day incubation were extended for a total of 14 days. Blood was then subcultured to Columbia blood agar (BA), chocolate agar (CHOC), MacConkey agar (MAC), and Brucella blood agar (BBA) (Thermo Fisher Scientific-Oxoid, Nepean, ON) plates and incubated anaerobically at $35^{\circ} \mathrm{C}$ for 48 hours before examination. However, no growth occurred on 


\section{CLINICAL HISTORY}

- Oct 19: Presents to Hospital with aortic and mitral valve vegetations. Started on Vancomycin, Ceftriaxone. - Oct 26: 10 teeth extracted. - Oct 27: Cardiovascular surgery.

- Nov 2: Discharged from hospital. - Nov 6: Returns to hospital with chills and dizziness. Diagnosed with tamponade, no evidence of new focal infection.

-Dec 8: Completes 6 weeks of parenteral Vancomycin, Ceftriaxone.

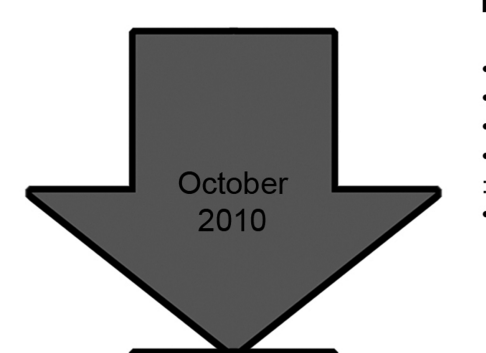

MICROBIOLOGY RESULTS

- Oct 19: PBC-1 = no growth, 4 days

- Oct 20: PBC $-2=$ no growth, 4 days

- Oct 22: $\mathrm{PBC}-3=$ no growth, 4 days

Oct 27: $\mathrm{CV}=$ no growth, 4 days

valve fungal/yeast = no growth, $4 \mathrm{wks}$

Oct 29: HIV test = negative
- Early Jan: Night sweats begin again. - Jan 24: Admitted to hospital for further investigations including: Repeat TEE, CT Chest, WBC nuclear imaging. All testing negative for new focal infection.

-Mar: Low grade clinical symptoms persist.

- Apr: Broad-range 16S PCR and sequencing performed.

- Apr 8: Antibiotics started.

-Apr 10: Resolution of symptoms.

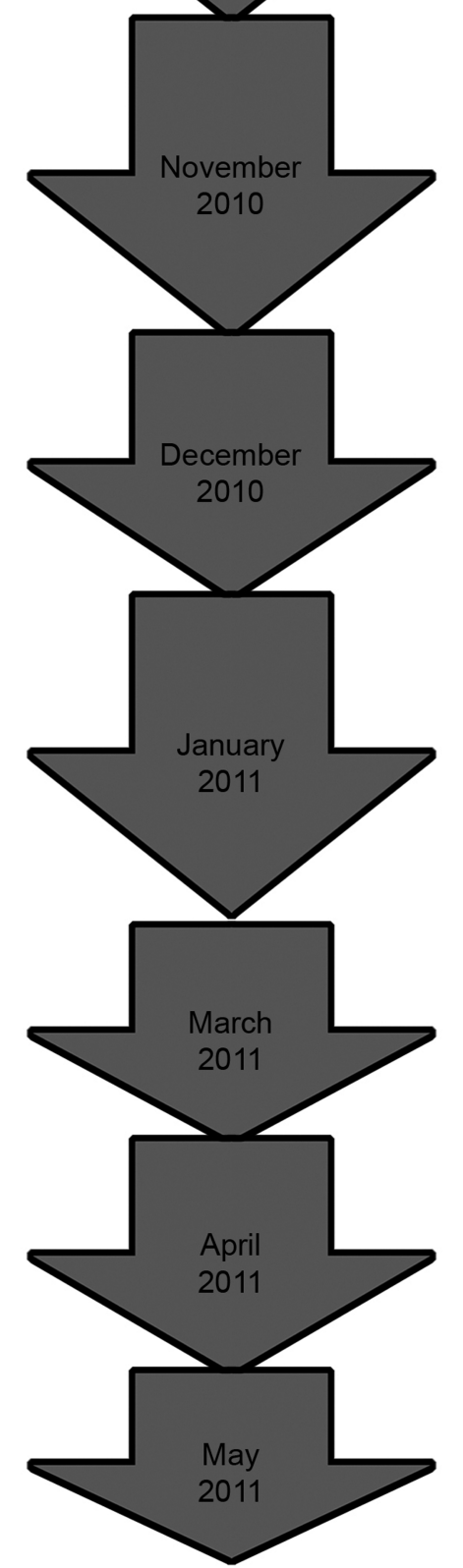

- Nov 7: PFC-4 = no growth, 4 days: pericardial fungal = no growth, $4 \mathrm{wks}$ - Nov 8: pericardial AFB smear $=$ negative $:$ Bartonella, $Q$ fever test $=$ negative - Nov 16: PBC-5 = no growth, 4 days
- Jan 24: PBC-6 = no growth, 4 days * - Jan 25: PBC-7 = no growth, 7 days

- Jan 28: PBC-8 = no growth, 4 days

- Jan 29: PBC-9 = no growth, 4 days

- Jan 31: PBC-10 = no growth, 4 days

- Apr 8: Blood-1 16S PCR = positive Bergeyella

Apr 13: Blood -2 16S PCR = positive Bergeyella

Apr 13: Blood -3 16S PCR = positive Bergeyella

- Apr 24: *16S PCR on PBC-6 = positive Bergeyella

- May 27: $\mathrm{PBC}-12$ = no growth 4 days $:$ Blood $16 \mathrm{~S} P C R=$ negative

Figure 1: Timeline of clinical history and microbiological results

$\mathrm{PBC}=$ peripheral blood culture; $\mathrm{VT}=$ valvular tissue; $\mathrm{PFC}=$ pericardial fluid culture; $\mathrm{ng}=$ no growth; TEE $=$ transesophageal echocardiogram; $\mathrm{CT}=$ computed tomography; $\mathrm{WBC}=$ white blood cell.

* Blood culture protocol includes both aerobic and anaerobic bottles. 
Table 1: Reported cases of Bergeyella sp. bacteremia with animal contacts (1),(8-11)

\begin{tabular}{lllll}
\hline Author (year) & Age/Sex & Comorbidities & Animal contact & Treatment \\
\hline Noell et al (1989) (8) & $80 / \mathrm{F}$ & Diabetes, bed sores & Cat & Cefotaxime \\
Montejo et al (2001) (1) & $33 / \mathrm{M}$ & None & Dog bite & Amoxicillin, clavulanic acid \\
Kivinen et al (2003) (9) & $77 / \mathrm{F}$ & Diabetes, steroid use & Cat & Cefuroxime \\
Beltran et al (2006) (10) & $44 / \mathrm{M}$ & None & Ingestion of goat blood & Ciprofloxacin \\
Lin et al (2007) (11) & $73 / \mathrm{M}$ & Cirrhosis, cancer & Dog & Cefazolin, gentamicin \\
\hline
\end{tabular}

BA, CHOC, MAC, or BBA plates, even with extended incubation under the same conditions for another 24 hours.

Blood samples from the patient, either an aliquot from previously collected blood culture bottles or drawn separately into an EDTA tube, were stored by the clinical laboratory at $-80^{\circ} \mathrm{C}$. Stored samples were sequentially tested using a laboratory-developed broad-range 16S PCR assay adapted from Bosshard et al (3). Briefly, DNA was extracted from blood culture bottle samples using PrepMan Ultra sample preparation reagent (Life Technologies, Inc, Thermo Fisher Scientific, Burlington, ON) or from whole blood EDTA using the QIAmp DNA Mini Kit Blood and Body Fluid protocol (Qiagen Inc, Germantown, MD). Broad-range 16S PCR was performed with primers BAK$11 \mathrm{w}$ and $\mathrm{BAK}-2$, where $\mathrm{M}=\mathrm{A} / \mathrm{C}$ and $\mathrm{H}=\mathrm{A} / \mathrm{C} / \mathrm{T}$ according to the conditions described previously (3). Bands were gel-purified with QIAQuick Gel Extraction Kit (Qiagen Inc, Germantown, MD) and re-amplified with BAK-11w and BAK-533r (5'-TTA CCG CGG CTG CTG GCAC-3') for 30 cycles. Amplification was confirmed by gel electrophoresis and semi-nested PCR product purified by QIAQuick PCR Purification Kit (Qiagen) or Exo-SAP-it (Thermo Fisher Scientific-Affymetrix Inc, Santa Clara, CA).

The amplicons were further purified with the Big Dye Xterminator Purification Kit (Life Technologies) and were sequenced on the ABI Prism 3130xl genetic analyzer (Thermo Fisher Scientific-Applied Biosystems Inc, Foster City, CA). A BLAST search against the IDNS Bacteria database (SmartGene IDNS, Lausanne, Switzerland) gave an identity score (99.8\%) for Bergeyella spp oral taxon 422 in four sequentially collected blood samples.

\section{Phylogenetic analysis}

A total of 18 partial $16 \mathrm{~S}$ rDNA sequences were analyzed: peripheral blood culture sample \#6 (PBC-6), 3 sequences amplified from whole EDTA blood (Blood-1, Blood-2, and Blood-3), and 14 publicly available sequences of Bergeyella spp and Prevotella buccalis ATCC 35310 (4).
Multiple sequence alignment was performed with MUSCLE version 3.8.31 (5), then inspected and trimmed with AliView version 1.15 (6). A maximum likelihood tree was inferred with FastTree version 2.1.4, with a distribution of 1,000 resampled trees under the generalized timereversible (GTR) model of evolution and the ShimodairaHasegawa test to calculate local support values (7).

\section{DIAGNOSIS}

Our patient had culture-negative endocarditis caused by Bergeyella spp. A BLAST search of the approximately 500-base-pair 16S sequence amplified from multiple blood samples against the SmartGene IDNS Bacteria database indicated that the most closely related species was Bergeyella spp oral taxon 422, and the overall identity score was $99.8 \%$. Phylogenetic analysis also confirmed that our blood culture isolates were identical to each other (1 SNP) and most closely related to the previously reported Bergeyella spp oral taxon $42216 \mathrm{~S}$ rDNA sequence (see Figure 2).

As Table 1 shows, cases of Bergeyella spp bacteremia in the literature are rare. Of the five cases of B. zoohelcum bacteremia reported to date, all five patients had a history of an animal bite or prolonged animal contact (1),(8-11).

Patients in other cases of Bergeyella spp identified in rare cases of cellulitis and tenosynovitis $(12,13)$ also had significant animal contact. In fact, only three cases documented in the literature do not specify a history of animal contact. Han et al identified Bergeyella spp in the amniotic fluid of a woman who gave birth to a premature infant; the same species had been identified through PCR 16s rDNA testing in her oral cavity, with unknown significance (14). Sohn et al (15) recently described two cases of infective endocarditis caused by novel Bergeyella spp which were initially misidentified as Brevundimonas spp. All of these cases suggest that Bergeyella species can be opportunistic pathogens for which treatment should be pursued in an attempt to avoid bacteremia and potential endocarditis. 


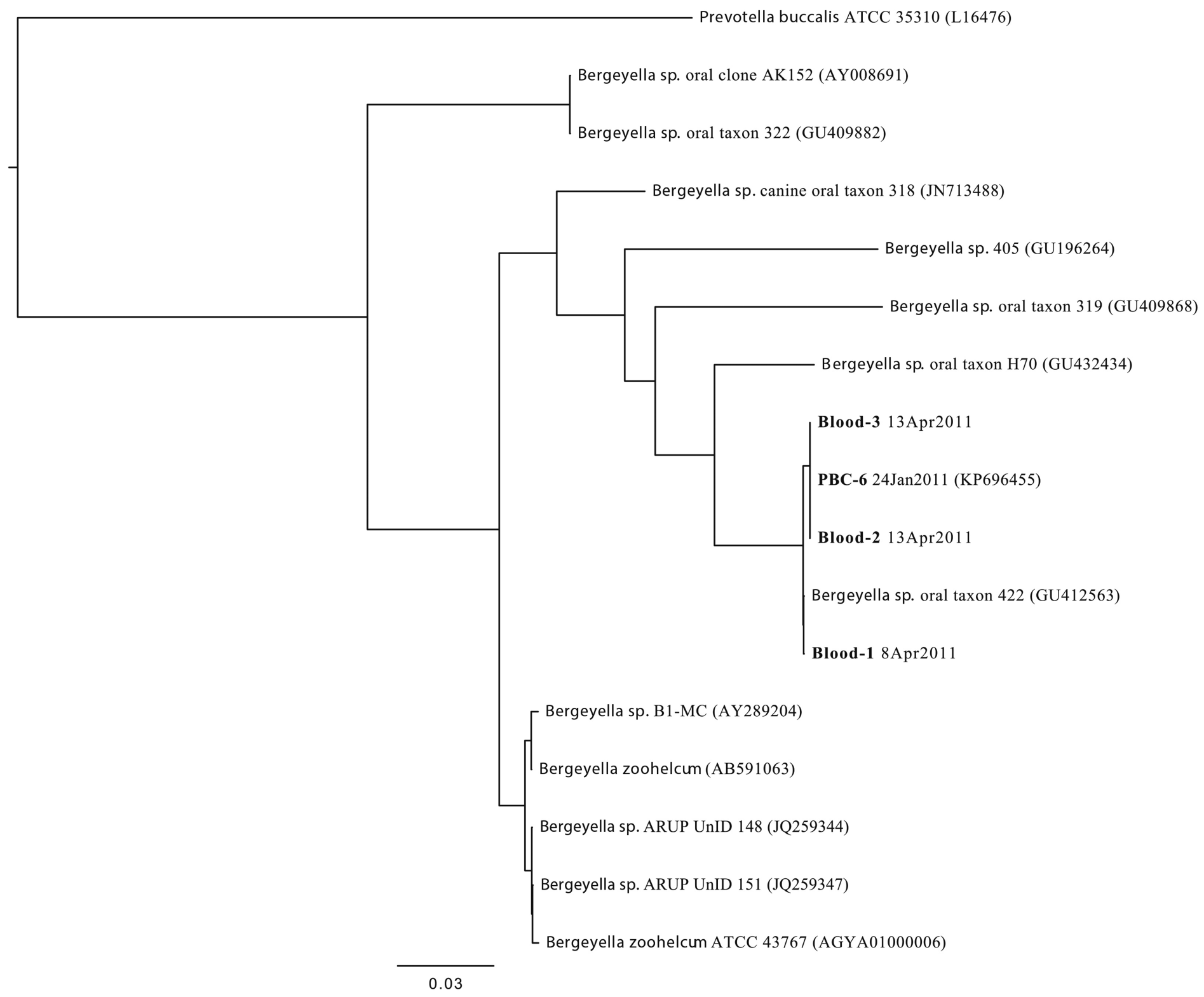

Figure 2: Bergeyella spp. phylogenetic tree

\section{DISCUSSION}

This is the first report of an oral taxon species of Bergeyella bacteremia causing endocarditis in a patient with severe periodontal infection and no prior animal contact. This case demonstrates that broad-range 16S rDNA sequencing is a powerful tool for detection and definitive molecular identification of important fastidious or non-cultivatable clinical isolates (16). Within the Bergeyella genus, there have now been multiple species assigned, most commonly Bergeyella zoohelcum, an organism most commonly identified as part of the normal oral flora of domesticated animals (17).
Although our patient did not give a clear history of animal contact, it should be noted that he had severe periodontal disease and underwent extensive oral surgery before his initial valve operation. The repeated isolation of this organism, along with correlating clinical symptoms, provides convincing data of an oral source in our particular case. Transient bacteremia following dental extraction may be more common than previously thought (18), and may not always reliably indicate a causative pathogen of an infectious disease syndrome. It is likely that our patient was colonized with Bergeyella spp oral taxon 422 and that, in conjunction with manipulation of his oral cavity through dental or routine oral hygiene practices, it led 
to a persistent bacteremia and subsequent endocarditis. Although the exact timing of infection cannot be determined, valve seeding most likely occurred sometime before his initial symptomatic presentation and/or during his dental procedure. Persistent infective endocarditis that involves biofilm on prosthetic material has a higher rate of relapse/recurrence. A large prognostic study of infective endocarditis in 420 patients showed that the risks of relapse (3.3\%) and recurrence (11.4\%) are significant, particularly for male patients and increased age (19). In that study, patients with prosthetic valve endocarditis also had a higher risk of relapse or recurrence requiring valve replacement. Given the age and sex of our patient and the presence of not only a prosthetic valve but also a large prosthetic aortic graft, therefore, it is not surprising that he returned with a relapse/recurrence of his symptoms despite an adequate course of initial antibiotic therapy.

Bergeyella spp may be difficult to detect using traditional culture methods (ie, it is non-cultivatable in blood culture bottle media or on routine nutrient media); in this case, it was detected only by $16 \mathrm{~s}$ rDNA amplification and sequencing, after considerable delay. If Bergeyella infection is suspected, then hydroxybutyrate agar $(20,21)$ and heart infusion medium containing $5 \%$ rabbit blood may be used to try to improve Bergeyella and Weeksella cultivation $(21,22)$.

Because our isolate was not cultivatable on standard bacterial media, data on antibiotic susceptibility were not available. However, Bergeyella spp have previously been reported to be susceptible to $\beta$-lactam antibiotics, chloramphenicol, gentamicin, and fluoroquinolones and to vary in susceptibility to trimethoprim-sulfamethoxazole and tetracycline $(22,23)$. Our review of the literature indicates that initial treatment with $\beta$-lactam antibiotics is a popular first-line choice (see Table 1).

In this case, we initially chose ceftriaxone and vancomycin for coverage against Staphylococcus aureus, viridans group streptococci, HACEK (Haemophilus spp, Aggregatibacter spp, Cardiobacterium hominis, Eikenella corrodens, and Kingella spp) organisms, and enterococci, in accordance with the 2015 American Heart Association guidelines on treatment of sub-acute culture-negative native valve endocarditis (24). By the time our patient suffered a relapse/ recurrence of symptoms, we had proof of Bergeyella spp bacteremia; we therefore added gentamicin for additional therapeutic coverage against this gram-negative aerobic bacillus. A fluoroquinolone was not used because of heightened concern about prolonging the QT interval due to the potential interaction with the patient's cardiac drugs (25).
Management strategies for relapsed infective endocarditis are not included in the AHA/IDSA treatment guidelines, beyond re-testing the susceptibilities of isolated causative pathogens, which was not possible in our situation.

There are no current guidelines on first-line antibiotic treatment in the context of previous Bergeyella spp infections. There has been limited research into the prevalence of Bergeyella spp in human oral cavities, but published data from Han et al (14) would suggest that Bergeyella is more prevalent than previously known. Is it possible that the association between dental disease and endocarditis is underreported because of less sensitive testing methods for previously unrecognized or difficult to detect organisms? In future cases of endocarditis, it may be useful to collect gingival swabs for molecular microbial community analysis to determine whether the causative organism is present as part of the patient's normal oral flora. In addition, we should try to culture Bergeyella from blood samples identified by molecular testing, in order to perform antibiotic susceptibility testing. More detailed information is needed about the usual susceptibility profile of this organism before evidence-based changes can be made to the current treatment guidelines to ensure coverage for Bergeyella.

In this case, the diagnosis was made because there was a high degree of clinical suspicion of ongoing infection, given the patient's persistent clinical symptoms. The ability to store and analyze previously drawn samples was what enabled us to make the diagnosis, and this should be emphasized to others dealing with persistent infective endocarditis symptoms in a patient with negative traditional blood culture results.

CONTRIBUTORS: All authors participated in conceiving and designing the study, interpreting the data, and critically revising the manuscript. Drs Clark and Chow acquired the data. Drs Clark, Parkins, and Church analyzed the data. Dr Clark drafted the manuscript that all authors reviewed and revised. All authors approved the final version accepted for publication.

ACKNOWLEDGEMENTS: Molecular assays were performed by the Laboratory Specialist, Molecular Microbiology Laboratory, Calgary Laboratory Services (DLC/ BLC).

FUNDING: No funding was received for this work.

DISCLOSURES: The authors have nothing to disclose. 


\section{REFERENCES}

1. Montejo M, Aguirrebengoa K, Ugalde J, Lopez L, Saez Nieto JA, Hernández JL. Bergeyella zoohelcum bacteremia after a dog bite. Clin Infect Dis. 2001;33(9):1608-9. https://doi.org/10.1086/322724. Medline:11588704.

2. Dewhirst FE, Chen T, Izard J, et al. The human oral microbiome. J Bacteriol. 2010;192(19):5002-17. https://doi.org/10.1128/JB.00542-10.

Medline:20656903.

3. Bosshard PP, Kronenberg A, Zbinden R, Ruef C, Böttger EC, Altwegg M. Etiologic diagnosis of infective endocarditis by broad-range polymerase chain reaction: a 3-year experience. Clin Infect Dis. 2003;37(2):167-72. https://doi.org/10.1086/375592. Medline:12856207.

4. Quast C, Pruesse E, Yilmaz P, et al. The SILVA ribosomal RNA gene database project: improved data processing and web-based tools. Nucleic Acids Res. 2013;41(Database issue):D590-6. https://doi.org/ 10.1093/nar/gks1219. Medline:23193283

5. Edgar RC. MUSCLE: multiple sequence alignment with high accuracy and high throughput. Nucleic Acids Res. 2004;32(5):1792-7. https://doi.org/10.1093/ nar/gkh340. Medline:15034147.

6. Larsson A. AliView: a fast and lightweight alignment viewer and editor for large datasets. Bioinformatics. 2014;30(22):3276-8. https://doi.org/10.1093/ bioinformatics/btu531. Medline:25095880.

7. Price MN, Dehal PS, Arkin AP. FastTree 2approximately maximum-likelihood trees for large alignments. PLoS One. 2010;5(3):e9490. https:// doi.org/10.1371/journal.pone.0009490. Medline:20224823.

8. Noell F, Gorce MF, Garde C, Bizet C. Isolation of Weeksella zoohelcum in septicaemia. Lancet. 1989;2(8658):332. https://doi.org/10.1016/S01406736(89)90518-7. Medline:2569133.

9. Kivinen PK, Lahtinen MR, Ruotsalainen E, Harvima IT, Katila ML. Bergeyella zoohelcum septicaemia of a patient suffering from severe skin infection. Acta Derm Venereol. 2003;83(1):74-5. Medline:12636038.

10. Beltran A, Bdiiwi S, Jani J, Recco RA, Go EE, Zaman MM. A case of Bergeyella zoohelcum bacteremia after ingestion of a dish prepared with goat blood. Clin Infect Dis. 2006;42(6):891-2. https://doi.org/10.1086/ 500457. Medline:16477574.

11. Lin WR, Chen YS, Liu YC. Cellulitis and bacteremia caused by Bergeyella zoohelcum. J Formos Med Assoc.
2007;106(7):573-6. https://doi.org/10.1016/S09296646(07)60008-4. Medline:17660147.

12. Isotalo PA, Edgar D, Toye B. Polymicrobial tenosynovitis with Pasteurella multocida and other gram negative bacilli after a Siberian tiger bite. J Clin Pathol. 2000;53(11):871-2. https://doi.org/10.1136/ jcp.53.11.871. Medline:11127273.

13. Shukla SK, Paustian DL, Stockwell PJ, et al. Isolation of a fastidious Bergeyella species associated with cellulitis after a cat bite and a phylogenetic comparison with Bergeyella zoohelcum strains. J Clin Microbiol. 2004;42(1):290-3. https://doi.org/10.1128/ JCM.42.1.290-293.2004. Medline:14715767.

14. Han YW, Ikegami A, Bissada NF, Herbst M, Redline RW, Ashmead GG. Transmission of an uncultivated Bergeyella strain from the oral cavity to amniotic fluid in a case of preterm birth. J Clin Microbiol. 2006;44(4):1475-83. https://doi.org/10.1128/ JCM.44.4.1475-1483.2006. Medline:16597879.

15. Sohn KM, Huh K, Baek J-Y, et al. A new causative bacteria of infective endocarditis, Bergeyella cardium sp. nov. Diagn Microbiol Infect Dis. 2015;81(3):213-6. https://doi.org/10.1016/j.diagmicrobio.2014.12.001. Medline:25544000.

16. Wellinghausen N, Kochem AJ, Disqué C, et al. Diagnosis of bacteremia in whole-blood samples by use of a commercial universal 16S rRNA gene-based PCR and sequence analysis. J Clin Microbiol. 2009;47(9):2759-65. https://doi.org/10.1128/ JCM.00567-09. Medline:19571030.

17. Holmes B, Steigerwalt AG, Weaver RE, Brenner DJ. Weeksella zoohelcum sp nov (formerly group IIj), found in human clinical specimens. Syst Appl Microbiol. 1986;8(3):191-6. https://doi.org/10.1016/ S0723-2020(86)80076-5.

18. Mansur AJ, Dal Bó CMR, Fukushima JT, Issa VS, Grinberg M, Pomerantzeff PM. Relapses, recurrences, valve replacements, and mortality during the longterm follow-up after infective endocarditis. Am Heart J. 2001;141(1):78-86. https://doi.org/10.1067/ mhj.2001.111952. Medline:11136490.

19. Benítez-Páez A, Álvarez M, Belda-Ferre P, Rubido S, Mira A, Tomás I. Detection of transient bacteraemia following dental extractions by $16 \mathrm{~S}$ rDNA pyrosequencing: a pilot study. PLoS One. 2013;8(3):e57782. https://doi.org/10.1371/ journal.pone.0057782. Medline:23469240.

20. Bernardet JF, Nakagawa Y, Holmes B. Proposed minimal standards for describing new taxa of the family Flavobacteriaceae and emended description of 
the family. Int J Syst Evol Microbiol. 2002;52(Pt 3):1049-70. Medline:12054224.

21. Hugo CJ, Bruun B, Jooste PJ. The genera Bergeyella and Weeksella. In: Dworkin M, Falkow S, Rosenberg E, Schleifer KH, Stackebrandt E, editors. The prokaryotes. Vol. 7. New York: Springer; 2006. p 5328. https://doi.org/10.1007/0-387-30747-8_18.

22. Dees SB, Moss CW, Hollis DG, Weaver RE. Chemical characterization of Flavobacterium odoratum,

Flavobacterium breve, and Flavobacterium-like groups IIe, IIh, and IIf. J Clin Microbiol. 1986;23(2):267-73. Medline:3700614.

23. Vay CA, Almuzara $\mathrm{MN}$, Rodríguez $\mathrm{CH}$, et al. ["In vitro" activity of different antimicrobial agents on gram-negative nonfermentative bacilli, excluding
Pseudomonas aeruginosa and Acinetobacter spp]. Rev Argent Microbiol. 2005;37(1):34-45.

Medline:15991478.

24. Baddour LM, Wilson WR, Bayer AS, et al. Infective endocarditis in adults: diagnosis, antimicrobial therapy, and management of complications: a scientific statement for healthcare professionals from the American Heart Association. Circulation. 2015;132(15):1435-86. https://doi.org/10.1161/ CIR.0000000000000296. Medline:26373316.

25. Rubinstein E, Camm J. Cardiotoxicity of fluoroquinolones. J Antimicrob Chemother. 2002;49(4):593-6. https://doi.org/10.1093/jac/ 49.4.593. Medline:11909831. 